\title{
Análisis del Perfil Antropométrico de Jugadores de la Selección Brasileña de Voleibol Infanto Juvenil
}

\author{
Analysis of Anthropometrical Profile of Brazilian Junior Volleyball Team
}

"Cláudio Luís Toledo Fonseca; **Paula Roquetti Fernandes \& ****José Fernandes Filho

\begin{abstract}
FONSECA, C. L. T.; FERNANDES, R. P. \& FERNANDES FILHO, J. Análisis del perfil antropométrico de jugadores de la selección brasileña de voleibol infanto juvenil. Int. J. Morphol., 28(4):1035-1041, 2010.

RESUMEN: El voleibol brasileño es reconocido por su excelencia en el ámbito internacional. Las victorias en las categorías de base permiten que jóvenes jugadores talentosos lleguen a las selecciones nacionales. Una herramienta importante en el proceso de selección de jóvenes atletas es la antropometría que evalúa la forma y tamaño de los jugadores. Esta investigación verificó las características antropométricas de los jugadores de la selección infanto juvenil de Brasil. Se evaluaron 16 jugadores con edad de 16,8 $\pm 0,58$ años del sexo masculino convocados para el Campeonato Sudamericano de 2006, utilizando el protocolo de Lohman para verificar la percentaje de grasa y el Somatotipo de Heath \& Carter de acuerdo con la posición dentro del campo de juego: colocador (COL) (n=3), libero $(\mathrm{LIB})(\mathrm{n}=2)$, central $(\mathrm{CEN})(\mathrm{n}=4)$, punta-receptor $(\mathrm{PR})(\mathrm{n}=4)$ y Opuesto $(\mathrm{OP})(\mathrm{n}=3)$. Fue utilizada la estadística descriptiva para la caracterización antropométrica y la ANOVA con post hoc de Tukey para observación de las diferencias entre las posiciones de los jugadores. Fueron observadas diferencias significativas en la masa corporal y estatura entre LIB y CEN, y entre LIB y OP. Sin embargo, no fueron observadas diferencias entre las posiciones de juego en porcentaje de grasa y componentes de lo somatotipo. Esto apunta para una selección de atletas que busca jugadores altos y lineales independiente de la función de campo. La única posición que presentó diferencias fue el LIB.
\end{abstract}

PALABRAS CLAVE: Antropometría; Composición corporal; Somatotipo; Voleibol.

\section{INTRODUCCIÓN}

Actualmente, Brasil ocupa un lugar prominente en el escenario internacional gracias a la conquista de varios títulos tanto en adulto como en las categorías de base, masculino y femenino. Mantener esta posición de liderazgo requiere un trabajo duro, dedicación, investigación y un programa de detección y promoción de talentos organizado y ejecutado con seriedad sobre la base de los procedimientos científicos.

La selección de jóvenes talentos en el voleibol permitirá la formación de reservas deportivas capaces de suplir los equipos adultos en las principales competiciones internacionales. Sin embargo, es necesario establecer indicadores de desempeño para guiar el proceso de selección de los deportes.
Los parámetros de selección deportiva pueden incluir evaluaciones antropométricas, sicológicas, pruebas de rendimiento físico, entre otras posibilidades para observar las variables de rendimiento (Fernandes Filho, 2003a). Los resultados de estas verificaciones cuando son observados en los más destacados atletas del deporte permiten identificar las características de un "modelo" que dirija el proceso de selección de talentos. No obstante, también es necesario observar las "variables intermedias", es decir, las puntuaciones obtenidas por los deportistas más calificados en estas pruebas de control durante el proceso de entrenamiento a largo plazo (Filin \& Volkov, 1998).

Las medidas antropométricas permiten comprobar los aspectos relacionados al tamaño y la forma de un individuo,

\footnotetext{
* Universidad Autónoma de Asunción - UAA - Asunción - Paraguay. Centro Universitário de Barra Mansa - UBM - Barra Mansa - Brasil.

** Centro de Excelência em Avaliação Física - CEAF - Brasil.

**** Departamento de Jogos / EEFD - UFRJ - Rio de Janeiro - Brasil.

LABIMH-UFRJ/CNPq
} 
lo que propicia el análisis de variables como masa corporal, estatura, composición corporal e incluso el tipo físico (Fernandes Filho, 2003b). Algunas características como estatura elevada (Gualdi-Russo \& Zaccagni, 2001) asociada con un perfil ecto-mesomorfórfico (Heath \& Carter, 1990) puede contribuir a aumentar la altura de la amplitud de las acciones de ataque y bloqueo lo que propicia ventajas para los atletas de voleibol.

Los indicadores antropométricos de los jugadores de voleibol masculino de adultos (Heimer et al., 1988, Heath \& Carter; Gualdi-Russo \& Zaccagni; Ciccarone et al. 2008, Flores et al., 2009), juvenil y infanto juvenil (Ciccarone et al., 2005; Duncan et al., 2006; Gabbett \& Georgieff, 2007) han sido presentados en la literatura para que permitan identificar las características de los atletas de alto rendimiento y de los equipos intermedios formados por jugadores jóvenes de esta modalidad.

Sin embargo, pocos estudios (Gualdi-Russo \& Zaccagni; Ciccarone et al.; Duncan et al.) han informado las diferencias antropométricas entre las posiciones técnico tácticas de los atletas de voleibol. Por lo tanto, este estudio busca determinar las características antropométricas de los atletas de la selección brasileña infanto juvenil de voleibol masculino de acuerdo con la función en la cancha.

Se cree que los datos obtenidos en este estudio servirán como parámetros para guiar el proceso de selección deportiva de atletas jóvenes de voleibol en lo que se refiere a variables antropométricas.

\section{MATERIAL Y MÉTODO}

Este estudio se caracterizó por un delineamiento transversal, descriptivo y comparativo. La muestra se seleccionó deliberadamente para estar compuesta por los 16 atletas invitados para la selección brasileña de voleibol infanto juvenil masculino de 2006, durante la preparación para el Campeonato Sudamericano de este deporte, del cual fue campeón. Los atletas tenían 16,8 \pm 0,58 años, y fueron evaluados en las instalaciones del Centro de Excelencia del Voleibol de Saquarema-RJ. Todos los atletas fueron voluntarios, autorizados por sus responsables y por el Comité Técnico para participar en las pruebas propuestas.

Las medidas antropométricas que se registraron fueron: la masa corporal, estatura, pliegues cutáneos: de tríceps, subescapular, supraespinoso y pantorrilla; circunferencias: de brazo "forzado" y pantorrilla; diámetros óseos: húmero y fémur. Para esta colección se utilizó una balanza Filizola, un estadiómetro Sanny un compás de espesor de pliegue cutáneo Lange, una cinta métrica y compás de espesor antropométrico de Sanny. Todas las medidas se obtuvieron mediante un único de conformidad con las directrices de ISAK, presentando coeficiente intra clase para a validad teste-reteste e error típico de: 0,99 e 0,3\% para masa corporal; 0,99 e $0,2 \%$ para la estatura; 0,97 e $3,0 \%$ para las pliegue cutáneas; 0,98 e 1,0\% para los diámetros óseos; e 0,99 y $0,5 \%$ para las circunferencias.

El cálculo del porcentaje de grasa utiliza el protocolo de Lohman (1986) citado por Fernandes Filho (2003b). Esta ecuación es adecuada para estimar la grasa corporal en niños y adolescentes de 6 hasta 17 años.

Las medidas de somatotipo se obtuvieron por el método somatotipológico de Heath \& Carter, que permite un estudio preciso del tipo físico ideal de cada deporte; es una excelente herramienta para utilizarse en el descubrimiento de talentos, además de permitir un continuo acompañamiento de los componentes somatotípicos durante una temporada competitiva. Éste posee un $r=0,98$, convirtiéndose así en un método seguro de evaluación (Heath \& Carter).

El análisis estadístico utilizó el test de Shapiro-Wilk para verificar la normalidad de los datos, que destacó el uso de técnicas de parámetros. En la descripción de los perfiles de los atletas se adoptó como medida de tendencia central, el promedio; como índices de dispersión: la desviación (DP) y el coeficiente de variación $(\mathrm{CV})$; relatándose también valores mínimos (Min) y máximos (Max) para variables de carácter continuo (peso, altura, porcentaje de grasa corporal y puntuaciones somatotípicas), y la distribución de frecuencias para las variables de naturaleza discreta (clasificación de somatotipo). Este procedimiento fue adoptado para caracterizar el grupo de atletas de acuerdo a la función en la cancha, divididos en: colocador (COL), libero (LIB), central (CEN) de punta-receptor (PR) y opuesto (OP).

Para identificar las diferencias entre las posiciones técnico tácticas en las variables dependientes se utilizaron los recursos de la estadística de inferencia, con el ANOVA (análisis de variación), con post-hoc de prueba de Tukey, y el nivel de significación de $\mathrm{p}<0,05$ (Callegari-Jacques, 2006). Todos los cálculos estadísticos se realizaron con el programa SPSS 16.0 para Windows.

\section{RESULTADOS}

Los resultados de los parámetros antropométricos se muestran en las Tablas I, II, III, que constituyen la puntua- 
ción observada en las variables, de peso, altura, porcentaje de grasa corporal y el somatotipo de los atletas de la selección brasileña infanto juvenil masculina.

Los resultados en la Tabla I muestran que la posición técnico y táctica que presentó atletas con masa corporal y estatura más elevada fue (CEN), seguida de (OP), mientras que el atleta más liviano y con estatura menor fue (LIB). Se registraron diferencias significativas en la masa corporal entre (LIB) y (CEN) $(\mathrm{p}=0,008)$, y (LIB) y (OP) $(\mathrm{p}=0,025)$ y en la altura también entre (LIB) y $(\mathrm{CEN})(\mathrm{p}=0,017)$, y (LIB) y $(\mathrm{OP})(\mathrm{p}=0,042)$. No se encontraron diferencias entre las otras funciones del juego.

La Tabla II presenta los resultados de porcentaje de grasa de los atletas de la selección brasileña infanto juvenil masculina que indicó para clasificación de las puntuaciones del grupo en general considerarse "adecuado" (10\% a 20\%) por edad y sexo según cuadro normativo proporcionado por Fernandes Filho (2003b). Fue verificado también que (CEN) y (OP) tenía los valores más altos entre las posiciones técni- co tácticas, y (LIB) tenían las puntuaciones más bajas. No obstante, no hubo diferencias significativas entre las funciones de juego.

La Tabla III muestra los datos relativos a las puntuaciones de los componentes somatotípicos y su clasificación. Se encontró que el $75 \%$ de todos los atletas independiente de la función en la cancha, presentaron el elemento ectomorfo. Esto demuestra la presencia de una alta concentración de individuos con elevada linealidad. No hubo diferencias significativas entre ninguna de las posiciones del juego.

\section{DISCUSIÓN}

Los resultados de la masa corporal de los atletas en este estudio fueron más altos en todas las funciones en la cancha, excepto (LIB), comparados con atletas juveniles de Inglaterra (Duncan et al.): (LEV) $(71,2 \pm 9,3 \mathrm{~kg}),(\mathrm{PR})(77,9$ $\pm 8,4 \mathrm{~kg}),(\mathrm{CEN})(77,6 \pm 5,9 \mathrm{~kg}), \mathrm{y}(\mathrm{OP})(71,3 \pm 9,2 \mathrm{~kg}), \mathrm{y}$ de

Tabla I. Caracterización de peso corporal y la altura de los atletas de la selección brasileña infanto juvenil masculina de acuerdo con la posición técnica y táctica.

\begin{tabular}{cccccccccccc}
\hline & \multicolumn{9}{c}{ Masa Corporal } & \multicolumn{4}{c}{ Est atura } \\
\cline { 2 - 11 } n & LEV & LIB & CEN & PR & OP & LEV & LIB & CEN & PO & OP \\
\cline { 2 - 11 } & 3 & 2 & 4 & 4 & 3 & 3 & 2 & 4 & 4 & 3 \\
Media* & 80,6 & $71,1 \#$ & $90,0 \#$ & 84,2 & $87,7 \#$ & 193,7 & $185,8 \#$ & $200,3 \#$ & 196,1 & $198,8 \#$ \\
DP & 0,32 & 7,00 & 5,20 & 6,36 & 3,24 & 3,79 & 3,18 & 3,20 & 5,98 & 3,55 \\
Min* & 80,4 & 66,1 & 83,3 & 76,8 & 85,0 & 191,0 & 183,5 & 198,0 & 189,0 & 195,0 \\
Máx* & 81,0 & 76,0 & 95,9 & 89,8 & 91,3 & 198,0 & 188,0 & 205,0 & 202,0 & 202,0 \\
CV(\%) & 0,4 & 9,9 & 5,8 & 7,6 & 3,7 & 2,0 & 1,7 & 1,6 & 3,0 & 1,8 \\
\hline
\end{tabular}

* Valores en kg de masa corporal, y cm de altura. $(\mathrm{LEV})=$ colocador; $(\mathrm{LIB})=$ liberal; $(\mathrm{CEN})=$ central; $(\mathrm{PO})=$ punta; $(\mathrm{OP})=$ opuesto. \# Diferencias significativas $\mathrm{p}<0,05$ entre (LIB) y (CEN); (LIB) y (OP) en la masa corporal estatura. \# Diferencias significativas $p<0,05$ entre (LIB) y (CEN), (LIB) y (OP) en la altura del cuerpo.

Tabla II. Caracterización del porcentaje de grasa de los atletas de la selección brasileña infanto juvenil masculina de acuerdo con la posición técnico y táctica.

\begin{tabular}{cccccc}
\hline & LEV & LIB & CEN & PR & OP \\
\hline $\mathbf{n}$ & 3 & 2 & 4 & 4 & 3 \\
Media* & 14,3 & 14,1 & 18,3 & 14,6 & 16,2 \\
DP & 0,7 & 4,2 & 3,5 & 3,9 & 2,7 \\
Mín* & 13,6 & 11,2 & 13,6 & 9,6 & 13,1 \\
Máx* & 14,9 & 17,1 & 22,0 & 18,7 & 17,9 \\
CV(\%) & 4,9 & 29,7 & 19,1 & 27,0 & 16,5 \\
\hline
\end{tabular}

$(\mathrm{LEV})=$ colocador $(\mathrm{LIB})=$ liberal $;(\mathrm{CEN})=$ central $;(\mathrm{PO})=$ punta;

$(\mathrm{OP})=$ opuesto.*Valores en porcentaje $(\%)$. 
Tabla III. Características del perfil somatotípico de atletas de voleibol infanto juvenil masculino de la selección brasileña, de conformidad con la posición técnica y táctica. * La clasificación somatotípica se realizó de acuerdo a las directrices (Fernandes Filhob).

\begin{tabular}{lccl}
\hline Posición & \multicolumn{1}{c}{ Puntuación } & \multicolumn{1}{c}{ Categoría * } & Distribución de frecuencias \\
\hline Colocador $(\mathrm{n}=3)$ & $2,3 \pm 0,3-3,0 \pm 0,7-4,2 \pm 0,7$ & Ecto-mesomórfico & $\begin{array}{l}66,6 \% \text { Ectomorfo balanceado } \\
33,3 \% \text { Ectomorfo mesomorfo }\end{array}$ \\
& & & $100 \%$ Ecto-mesomórfico \\
Libero $(\mathrm{n}=2)$ & $2,4 \pm 0,5-3,1 \pm 0,2-4,3 \pm 0,5$ & Ecto-mesomórfico & $25 \%$ Ectomorfo balanceado \\
Central $(\mathrm{n}=4)$ & $3,1 \pm 0,9-2,2 \pm 0,4-4,1 \pm 0,5$ & Ecto-endomórfico & $25 \%$ Ecto-endomórfico \\
& & & $25 \%$ Ecto-mesomórfico \\
& & & $25 \%$ Endomorfo-ectomorfo \\
& & & $50 \%$ Ectomorfo balanceado \\
Punta $(\mathrm{n}=4)$ & $2,4 \pm 0,5-2,7 \pm 1,7-4,2 \pm 0,6$ & Ectomorfo Balanceado & $25 \%$ Meso-ectomórfico \\
& & & $25 \%$ Ecto-endomórfico \\
& & & $33,3 \%$ Central \\
Opuesto $(\mathrm{n}=3)$ & $2,7 \pm 0,6-3,2 \pm 0,7-4,2 \pm 0,8$ & Ectomorfo Balanceado & $33,3 \%$ Ectomorfo balanceado \\
& & & $33,3 \%$ Ectomorfo mesomorfo \\
\hline
\end{tabular}

la selección juvenil italiana (Ciccarone et al., 2005): (COL) $(78,7 \pm 5,5 \mathrm{~kg}),(\mathrm{PR})(83,9 \pm 6,3 \mathrm{~kg}),(\mathrm{CEN})(84,3 \pm 4,9 \mathrm{~kg}), \mathrm{y}$ (OP) $(82,5 \pm 8,3 \mathrm{~kg})$. Sin embargo, Salem \& Zary (2004) encontraron valores más altos en todas las funciones de la cancha en los equipos juveniles de Brasil en 2000 y 2003: (COL) $81.7 \pm 5,13 \mathrm{~kg}$ y $80,2 \pm 0,8 \mathrm{~kg}$, (PR) $86.1 \pm 5,17 \mathrm{~kg}$ y $91,7 \pm$ $3,61 \mathrm{~kg}$; (CEN) 91,8 $\pm 3,91 \mathrm{~kg}$ y 93,2 $\pm 3,4 \mathrm{~kg}$; (PR) 94,4 \pm $5,52 \mathrm{~kg}$ y $96,6 \pm 6,05 \mathrm{~kg}$, y (LIB) 79,1 $\pm 1,48 \mathrm{~kg}$ y $83,8 \mathrm{~kg}$, respectivamente.

Los datos de los atletas evaluados muestran puntuaciones elevadas de masa corporal de acuerdo con el modelo internacional de la categoría juvenil, que se compone de individuos con edad más avanzada. No obstante, estos indicadores resultaron ser todavía insuficientes para los parámetros del voleibol brasileño de la categoría juvenil, lo que sugiere que estas personas deberán recibir entrenamientos que optimicen el aumento de masa muscular. Esta afirmación puede ser confirmada por los hallazgos de Zary \& Fernandes Filho, que muestran una tendencia de los atletas de las selecciones brasileñas masculinas de voleibol a presentar una masa delgada relativa cada vez más elevada hasta alcanzar la categoría de adultos.

Los resultados de la masa corporal de este estudio no corroboraron el estudio de Duncan et al. y Ciccarone et al. (2008), que no encontró diferencias entre las distintas posiciones técnicas y tácticas en atletas de la categoría juvenil, sin embargo, en estos dos estudios, (LIB) no se incluyó en el proceso de verificación de las medidas antropométricas. Ya Marques et al. (2009) encontró diferencias significativas entre (LIB) y todas las demás posiciones en los atletas adultos, a excepción de (COL).

A pesar de eso, los resultados obtenidos corroboran los datos facilitados por Ciccarone et al. que señalan (CEN) como los atletas con la masa corporal superior y (LIB) con menor peso corporal entre los puestos técnicos y tácticos. Sólo el estudio de Duncan et al. no muestra resultados similares, probablemente debido a la baja estatura encontrada por (CEN).

Las puntuaciones de los atletas de la selección brasileña infanto juvenil presentaron índices de estatura superiores a los atletas adultos, de acuerdo a la función en la cancha, cuando se consideran atletas italianos participantes en la Liga A1 y A2, informó Gualdi-Russo \& Zaccagni (COL) 185, $2 \pm$ 7,0 $\mathrm{cm}(\mathrm{PO}) 191,6 \pm 5,3 \mathrm{~cm}(\mathrm{CEN}) 196,6 \pm 5,1 \mathrm{~cm}(\mathrm{OP}) 194,7 \pm$ $5,1 \mathrm{~cm}$, y similar a los resultados del estudio de Ciccarone et al. (2008) (PR) 196,9 $\pm 3,9 \mathrm{~cm}$ y (CEN) $200,2 \pm 4,2 \mathrm{~cm}$, inferior a (LIB) $193,7 \pm 8,9 \mathrm{~cm}$ y hasta (LEV) $188,4 \pm 7,9 \mathrm{~cm}$.

Cuando se observaron las puntuaciones de los jóvenes deportistas, los datos de este estudio fueron inferiores a los de los atletas de la selección brasileña juvenil de voleibol masculina de 2000 y 2003 que han encontrado Salem \& Zary, a excepción de los levantadores (COL) 191,3 $\pm 4,11 \mathrm{~cm}$ y $186,55 \pm 0,55 \mathrm{~cm}(\mathrm{PR}) 198,64 \pm 5,18 \mathrm{~cm}$ y $201,25 \pm 3,66 \mathrm{~cm}$ (CEN) $202,17 \pm 3,19 \mathrm{~cm}$ y $204 \pm 2,29 \mathrm{~cm}(\mathrm{PR}), 203,88 \pm 5,95$ cm y $200,4 \pm 5,1 \mathrm{~cm}$ (LIB) $188 \pm 5,66 \mathrm{~cm}$ y $194 \pm 0,0 \mathrm{~cm}$. Sin embargo, Duncan et al. y Ciccarone et al. (2005) investigaron también la estatura de los atletas jóvenes de Inglaterra e Italia y se encontraron indicadores más bajos para todas las posiciones: (COL) $191 \pm 5,0 \mathrm{~cm}$ y 191,1 $\pm 3,8 \mathrm{~cm}$ (PR) 193, \pm 4,5 cm y $194,4 \pm 4,1 \mathrm{~cm}(\mathrm{CEN}) 187 \pm 3,6 \mathrm{~cm}$ y $198,2 \pm 4,1 \mathrm{~cm}$ (OP) $190 \pm 5,9 \mathrm{~cm}$ y $195,8 \pm 5,4 \mathrm{~cm}$, respectivamente.

Se pudo observar de nuevo que los atletas infanto juveniles brasileños tenían puntuaciones de altura de conformidad con los requisitos del escenario internacional de la categoría juvenil e incluso adulta, pero estos resultados todavía están por debajo de los conseguidos por los atletas brasileños de la categoría juvenil. No obstante, vale recordar que atletas de esta edad todavía poseen potencial para aumentar su estatura (Flores et al.). 
Tabla IV. Perfil del voleibol somatotípico de los equipos nacionales de Brasil, en diversas categorías.

\begin{tabular}{ccccc}
\hline Categoría & $\begin{array}{c}\text { Infantil-juvenil } \\
(\mathbf{2 0 0 6})^{*}\end{array}$ & $\begin{array}{c}\text { Infantil-juvenil } \\
(\mathbf{2 0 0 4 )} * *\end{array}$ & $\begin{array}{c}\text { Juvenil } \\
(\mathbf{2 0 0 3 )} * *\end{array}$ & $\begin{array}{c}\text { Adulta } \\
(\mathbf{2 0 0 4 )} * *\end{array}$ \\
\hline Endomorfía & $2,6 \pm 0,6$ & $2,4 \pm 0,5$ & $1,7 \pm 0,3$ & $2,6 \pm 0,6$ \\
Mesomorfía & $2,8 \pm 0,9$ & $3,4 \pm 0,7$ & $3,8 \pm 0,8$ & $4,2 \pm 1,6$ \\
Ectomorfía & $4,2 \pm 0,5$ & $3,8 \pm 0,7$ & $3,9 \pm 0,6$ & $2,6 \pm 0,8$ \\
Clasificación & Ectomorfo & Ectomorfo & Ectomorfo mesomorfo & Mesomorfo \\
somatotípica & balanceado & mesomorfo & & balanceado \\
\hline
\end{tabular}

* Datos obtenidos en este estudio. ** Datos obtenidos del estudio de Zary \& Fernandes Filho (2007).

Al considerar las diferencias entre posiciones técnicas y tácticas, los resultados fueron diferentes a los encontrados por Ciccarone et al. (2005), que identificó diferencias significativas sólo entre (COL) y (CEN) en atletas juveniles italianos, y Duncan et al. que no encontraron diferencias significativas en relación a la estatura de de los atletas juveniles británicos en las diversas posiciones técnicas y tácticas. Los estudios de atletas adultos mostraron diferencias significativas en la altura entre (COL), y todos los atacantes (CEN), (PR) y (OP) (Gualdi-Russo \& Zaccagni) y entre (CEN) y (LIB) y (CEN) y (LEV) (Ciccarone et al., 2008).

Teniendo en cuenta los datos observados en el estudio se puede afirmar que el proceso de selección de talentos en el voleibol de Brasil ha tratado de elegir a las personas que tienen una altura elevada independiente de la función, a excepción del (LIB). Se observó que, incluso (COL), presentó puntajes altos y (CEN) tenía valores superiores a todas las demás funciones de la cancha, atendiendo a las exigencias de la posición para el alto rendimiento.

$\mathrm{Al}$ analizar el porcentaje de grasa presentado por los atletas de este estudio, encontramos que estos resultados fueron superiores a los obtenidos en atletas juveniles brasileños $(8,3 \pm 0,9 \%)$ (Stanganelli et al., 2008) e italianos (10, $9 \pm$ 1,8\%) (Ciccarone et al., 2005), además de los atletas adultos italianos $(10,7 \pm 1,9 \%)$ (Ciccarone et al., 2008) y Iraníes $(10,45 \%)$ (Koozechian \& Nazen, 2002). No obstante, fueron más bajos que los puntajes de los atletas adultos de las selecciones de Venezuela (18,9 $\pm 3,2 \%)$, Uruguay $(20,4 \pm 2,8 \%)$, Paraguay $(20,2 \pm 2,7 \%)$, y Colombia $(20,6 \pm 0,8 \%)$, y de la selección juvenil de Chile (21,6 $\pm 4,6 \%)$ (Flores et al.).

Ya Salem \& Zary encontraron valores más bajos para los juveniles del equipo nacional en 2000 y 2003 en todas las funciones del juego: (COL) $(8,4 \pm 1,4 \%$ y $5,0 \pm 0,4 \%)$ (LIB) $(6,5 \pm 1,0 \%$ y $9,1 \%)$, (CEN) $(9,5 \pm 3,1 \%$ y $5,9 \pm 0,7 \%)$, (PR) $(6,2 \pm 2,0$ y el $5,7 \% \pm 1,3 \%)(\mathrm{OP})(9,7 \pm 1,1 \%$ y $7,0 \pm 0,4 \%)$. Sin embargo, estos resultados muestran la misma tendencia del estudio en cuestión, señalando (CEN) y (OP) como los atletas con el porcentaje de grasa más altos.
Estos resultados elevados en relación con las normas internacionales de las selecciones principales pueden estar relacionados con el hecho de que estos atletas tienen menor edad y tiempo de práctica que los atletas adultos y juveniles.

La influencia de la función técnica y táctica en relación con los parámetros de somatotipo fue reportada por Gualdi-Russo \& Zaccagni que describieron datos obtenidos en atletas adultos con valores de: (COL) $(2,4 \pm 0,7$ a $4,5 \pm 0,9$ $-2,8 \pm 0,8),(\mathrm{PR})(2,2 \pm 0,6$ a $4,3 \pm 0,9$ a $3,0 \pm 0,7)$, (CEN) $(2,0$ $\pm 0,6-4,0 \pm 1,0$ a $3,5 \pm 0,8)$ y (OP) $(2,2 \pm 0,6$ a $4,3 \pm 0,9$ a 3,1 $\pm 0,8)$, y verificaron la existencia de diferencias significativas entre (COL) y (CEN) para los 3 componentes, y entre (CEN) y (PR) para el componente ectomórfico. Ya Duncan et al. relataron índices somatotípicos para atletas de la selección juvenil inglesa, de acuerdo con la función en la cancha, de: (COL) $(2,6 \pm 0,9$ a $1,9 \pm 1,1$ a $5,3 \pm 1,2),(\mathrm{PR})(2,4 \pm 0,5$ a 2,6 $\pm 0,6$ a $4,6 \pm 0,8),(\mathrm{CEN})(2,2 \pm 0,8$ a $3,9 \pm 0,4$ a $3,6 \pm 0,7)$, (OP) $(2,3 \pm 0,8$ a $2,5 \pm 1,0$ a $5,1 \pm 1,1)$ y también se verificó la existencia de diferencias significativas entre los (CEN) y (COL) en relación con la ectomorfía y mesomorfía de estos atletas. Estos datos sugieren la existencia de diferencias entre los componentes del somatotipo en relación con la posición técnica y táctica, especialmente para (CEN), entre los atletas juveniles y adultos.

Sin embargo, los resultados de este estudio no encontraron diferencias entre ninguna posición técnico y táctica en relación con los componentes somatotípicos, hecho que apunta a una gran presencia de individuos con un perfil somatotípico ectomórfico, considerado adecuado para voleibol de alto rendimiento (Heath \& Carter), independiente de su función en la cancha, asociado a valores endomórficos (grasa) y mesomórficos (masa delgada relativa) inferiores.

No obstante, según Zary \& Fernandes Filho, hay una tendencia a aumentar las puntuaciones del componente mesomórfico y la disminución de las puntuaciones de ectomorfía de jugadores de voleibol de Brasil, durante el programa de capacitación a largo plazo. Estas mudanzas provienen de la fase de madurez, edad de los jugadores, el 
tipo de entrenamiento, género, nutrición y los aspectos raciales. Se observó esta tendencia en la Tabla IV, y se comprueba el aumento de la puntuación del componente ectomórfico de la selección de 2006 y 2004, lo que sugiere una preocupación en buscar atletas jóvenes más lineales, para cumplir los requisitos del voleibol internacional.

Se observó que los atletas infanto juveniles brasileños en Brasil presentan un gran potencial antropométrico para el escenario internacional, destacando la elevada estatura, un porcentaje de grasa corporal considerado "adecuado" asociado a una masa corporal dentro de los modelos de la modalidad, y un somatotipo de alta linealidad.

Los resultados de las medidas antropométricas de los atletas de este estudio que se presentaron en relación con los atletas juveniles brasileños son: inferiores para la masa corporal y estatura; superiores para el porcentaje de grasa; y con somatotipo que demuestra mesomorfía más baja; pueden ser considerados normales, visto que los atletas evaluados presentan menos edad, lo que los deja más sujetos a la influencia del proceso de crecimiento y desarrollo, además de un tiempo de práctica inferior al de los atletas más calificados, que los sitúa en las fases más precoces del programa de entrenamiento a largo plazo.

Es de destacar que el coeficiente de variación (CV) de las puntuaciones obtenidas en el análisis antropométrico es "bajo" ( $\mathrm{CV}=<20 \%)$ y apunta para la presencia de un grupo de jugadores de una misma función con características similares. Además, la ausencia de diferencias significativas entre las posiciones técnico tácticas en varias medidas observadas fue notoria, apuntando para un grupo de jugadores que tienen un tipo antropométrico bien definido, homogéneo, donde sólo (LIB) mostró perfil diferenciado de los demás.

Por lo tanto, podemos estar frente a un proceso de selección de talentos muy bien organizado, que busca jóvenes atletas con alto potencial antropométrico independientemente de su papel en la cancha y adecuado a las normas internacionales.

FONSECA, C. L. T.; FERNANDES, R. P. \& FERNANDES FILHO, J. Analysis of anthropometrical profile of Brazilian junior volleyball team. Int. J. Morphol., 28(4):1031-1034, 2010.

SUMMARY: Brazilian volleyball is recognized for its excellence in international tournaments. The victories in youth competition allow young talent players to participate on national teams. An important instrument in youth selection process is anthropometry that evaluates shape and body size of the athletes. This research verifies the anthropometrical characteristics of Brazilian junior volleyball players. Sixteen male players with 16,8 \pm 0,58 years old selected for 2006 South American Championship were evaluated, using Lohman protocols to access body fat, and Heath \& Carter somatotype according to players position: setters (SE) ( $\mathrm{n}=3$ ), libero (LIB) $(n=2)$, middle $(M I)(n=4)$, Outside $(O U)(n=4)$, Opposite $(O P)(n=4)$. Descriptive statistics to characterized the anthropometrical profile and ANOVA with Tukey's post hoc for detecting differences were used among players position. Significant differences for body mass and height between LIB and MI, and LIB and OP, were observed. However, no differences between player position were observed for percentage of body fat and somatotype components. This result lead for athlete selection that looks for tall and linear players independent of player position. The only position that showed a differents was LIB.

KEY WORDS: Anthropometry; Body composition; Somatotype; Volleyball.

\section{REFERENCIAS BIBLIOGRÁFICAS}

Callegari-Jaques, S. Bioestatística: princípios e aplicações. Porto Alegre, Artmed, 2006.

Ciccarone, G.; Croiser J.; Fontani, G.; Martelli, G.; Albert, A.; Zhang, L. \& Cloes, M. Comparison between player specialization, anthropometric characteristics and jumping ability in top-level volleybal players. Medicina Dello Sport, 61(1):29-43, 2008.

Ciccarone, G., Fontani, G; Albert, A.; Zhang, L. \& Cloes, M. Analisi delle caratteristiche antropometriche e delle capacitá di salto di giovani pallavolisti di alto livelo. Medicina Dello Sport, 58(1):1-15, 2005.
Duncan, M. J.; Woodfield, L. \& al-Nakeeb, Y. Anthropometric and physiological characteristics of junior elite volleyball players. Br. J. Sports Med., 40(1):649-51, 2006.

Fernandes Filho, J. Treinamento Desportivo: Descoberta de Talentos. Rio de Janeiro, Shape-CD Rom, 2003a.

Fernandes Filho, J. A prática da avaliação física. Rio de Janeiro, Shape, 2003b.

Filin, V. \& Volkov, V. Seleção de Talentos nos Desportos. Londrina, Midiograf, 1998. 
-Flores, A.; Rodriguez, F.; Gómez, F.; Arce, P.; Marincovich, D. \& Gutiérrez, O. Perfil antropométrico de jugadores profesionales de voleibol sudamericano. Int. J. Morphol. 27(1):53-7, 2009.

Gabbett, T. \& Georgieff, B. Physiological and anthropometric characteristics of Australian junior national, state, and novice volleyball players. J. Strength Cond. Res., 21(3):902-8, 2007.

Gualdi-Russo, E. \& Zaccagni, L. Somatotype, role and performance in elite volleyball players. J. Sports Med. Phys. Fitness, 41(2):256-62, 2001.

Heath, B. H. \& Carter, J. E. L. Somatotyping development and applications. New York, Cambridge University Press, 1990.

Heimer, S.; Misigoj, M. \& Medved, V. Some anthropological characteristics of top volleyball players in Yugoslavia. J. Sports Med. Phys. Fitness, 28(1):200-8, 1988.

Koozechian, H. \& Nazen, F. Comparison of characteristics of somatotype, physiological and chosen motor abilities between elite and beginner volleyball players. Int. J. Phys. Educ., 39(1):36-41, 2002.

Marques, M. C.; Tillaar, R.; Gabbett, T. J.; Reis, V. M.; \&González-Badillo, J. J. Physical fitness qualities of professional volleyball players: determination of positional differences. J. Strength Cond. Res., 23(4):1106-11, 2009.

Salem, M. \& Zary, J. C. F. Evolução do perfil somatotípico da seleção brasileira de voleibol masculino juvenil de 2000/2003. Rev. Educ. Fís., 128(1):41-51, 2004.

Stanganelli, L. C. R.; Dourado, A. C.; Oncken, P.; Mançan, S. \& Costa, S. C. Adaptations on jump capacity in brazilian volleyball players prior to the under-19 world championship. J. Strength Cond. Res., 22(3):741-9, 2008.

Thomas, J. R.; Nelson, J. K. \& Silverman, S. J. Métodos e técnicas em actividade física. Porto Alegre, Artmed, 2007.

Zary, J. C. F. \& Fernandes Filho, J. Identificação do perfil dermatoglífico e somatotípico dos atletas dovelibol masculino adulto, juvenil e infanto juvenil de alto rendimiento do Brasil. Rev. Bras. Ciencia Mov., 15(1):53-60, 2007.
Dirección para correspondencia:

Cláudio Luís Toledo Fonseca

Rua José Alves Pimenta, 1872

Matadouro

Barra do Piraí

Rio de Janeiro

BRASIL

Tel: 55(24) 2444-4572

55(24) $9817-6732$

Email: cltf5@yahoo.com.br

Recibido : 13-03-2010

Aceptado: 28-08-2010 
